\title{
Modeling of Polymer Erosion ${ }^{\dagger}$
}

\author{
Achim Göpferich and Robert Langer* \\ Department of Chemical Engineering, Building E25, Room 342, \\ Massachusetts Institute of Technology, Cambridge, Massachusetts 02139
}

Received March 23, 1993; Revised Manuscript Received May 12, 1993

\begin{abstract}
The erosion of bioerodible polymers depends on many factors including the polymer chain length, bond cleavage velocity, swellability, crystallinity, and water diffusivity in the polymer matrix. This multitude of parameters makes modeling of erosion difficult. Only a few models exist that describe morphological changes of polymers during erosion qualitatively. In the present approach the polymer matrix was represented as the sum of small individual polymer matrix parts. The factors that determine erosion were combined, and the erosion of each matrix piece was regarded as a random event. Once such a matrix piece had come into contact with water, an individual life expectation was assigned to it using Monte Carlo techniques. The proposed model can describe complicated phenomena such as changes in polymer matrix microstructure, movement of erosion fronts, creation of pores, and weight loss during erosion, yet it is simple and easy to use. For quantitative evaluations the model was fit to experimental data for weight loss and erosion front movement. The so obtained model constants proved to be useful for the prediction of independent parameters like the porosity of polymer matrices during erosion. This modeling approach may help broaden the understanding of the role of polymer erosion when considering bioerodible polymers in applications such as controlled drug delivery or tissue engineering.
\end{abstract}

\section{Introduction}

Biodegradable polymers have been used for a variety of applications including orthopedic implants, ${ }^{1}$ controlled release of drugs, ${ }^{2}$ and disposable plastics. ${ }^{3,4}$ All these applications benefit from the fact that the polymer "disappears" after providing a desired function. Two major processes are involved: the degradation of bonds between monomers in the polymer chains and the erosion of bulk polymer. ${ }^{5}$ The changes caused by erosion are macroscopically and microscopically visible. The importance of these changes is evident for drug-releasing biodegradable implants. The way an implant erodes will determine, for example, whether the drug is released immediately upon erosion or whether it must pass through diffusional layers or highly porous and tortuous networks. This release mechanism will in turn affect the drug pharmacokinetics, which is critical for determining drug performance.

For most polymers, erosion is a very complex process that depends on many factors including polymer degradation, swellability, crystallinity, hydrophobicity, polymer chain length, and water diffusivity. The multitude of parameters and the complexity of the individual processes involved in polymer erosion hinder the development of theoretical models that could be used for simulations, predictions, and better understanding of the process. There are currently only a few approaches for modeling polymer erosion. 6,7 Erosion was first described as a dissolution process $^{8}$ that affects only the polymer matrix surface; erosion, thereby, controls drug release. Later models described diffusion as a pseudo-steady-state process, while erosion is regarded as a first-order reaction ${ }^{9,10}$ or described by a constantly moving erosion front. ${ }^{11}$ To make models more flexible, the non-steady-state analysis of diffusion was introduced, and erosion was described by a moving front to which a constant velocity was assigned. ${ }^{12}$ Rather than describing erosion and diffusion, more recent models describe the simultaneous processes of degradation and diffusion. ${ }^{13}$ The main purpose of all these models is to explain the release of drugs from bioerodible polymers. By virtue of their one-dimensional character, however,

\footnotetext{
${ }^{\dagger}$ Dedicated to Dr. Maria Ott on the occasion of her 70th birthday.
}

- To whom correspondence should be addressed. they cannot explain microstructural changes of a polymer during erosion. One of the first two-dimensional models describes the release of drugs from matrix tablets consisting of a drug, polymer, and filler. ${ }^{14,15}$ Matrix cross-sections were represented by a two-dimensionalgrid, and unit cells of drug, polymer, or filler were assumed to dissolve at constant rates according to an algorithm which was originally applied to whole polymer matrices. ${ }^{8}$

Our erosion model followed this idea of the twodimensional representation of polymer matrices, which offers the simplest design that can describe structural changes. Rather than describing whole matrices as in current models, however, we concentrated on cutouts of polymer matrices to reduce the grid size. This was important to reduce the amount of involved calculations and was achieved by introducing appropriate boundary conditions. Periodic boundary conditions were used to account for the truncation of the grid orthogonally to the surface, and an insulation condition was used to account for the grid ending in the middle of the matrix. In contrast to the dissolution of drug particles, the application of surface detachment kinetics ${ }^{8,15}$ to the erosion of polymer was no longer possible. We decided, therefore, to take a new point of view and regarded the erosion of each small piece of polymer as a random event.

With our model, we attempted to describe the erosion of two biodegradable polyanhydrides, the erosion mechanism of which was recently reported. ${ }^{16}$ These polymers, p(CPP-SA) 20:80 and p(CPP-SA) 50:50, consisted of sebacic acid (SA) and 1,3-bis(p-carboxyphenoxy)propane (CPP) and differed only by the monomer ratio. There are two main morphological characteristics we intended to describe by the proposed model: (1) The thickness of matrices prepared from these polymers does not change for several days during erosion. (2) The erosion front that separates eroded from non-eroded polymer always moves parallel to the polymer matrix surface that is exposed to the buffer solution. These characteristics cannot be described by current models and, thus, were the target of the new modeling approach. A general algorithm was developed consisting of three consecutive steps: First, the two-dimensional grid was configured to describe the matrix prior to erosion. Next, the way erosion proceeds was 
defined. Finally, several functions such as erosion front velocity, matrix weight loss, and total porosity were defined that allow the calculation of parameters which are experimentally accessible.

The model constants for the two polymers were determined from the function for the velocity of the erosion front and from the function for matrix weight loss by fitting the model to experimental data. Once these constants were known, it was possible to predict the behavior of other parameters. As an example, the porosity of the matrices during erosion was predicted, and the results were compared with experimental data.

\section{Materials and Methods}

2.1. Materials. Sebacic acid (SA) and 1,3 -bis( $p$-carboxyphenoxy) propane (CPP) monomer as well as two copolymers, $\mathrm{p}(\mathrm{CPP}-$ SA) 20:80 and p(CPP-SA) 50:50, were obtained from Scios-Nova Pharmaceuticals, Baltimore, MD. The copolymers were prepared by melt polycondensation ${ }^{17}$ and differed by the weight ratio of monomers. Monobasic sodium phosphate and dibasic sodium phosphate were purchased in analytical grade from Mallinckrodt, Paris, KY.

2.2. Methods. Erosion of Polymer Matrix Disks. Cylindrically shaped polymer matrices $14 \mathrm{~mm}$ in diameter and 1.2 $\mathrm{mm}$ in height were prepared by melt casting ${ }^{18}$ and eroded in 10 $\mathrm{mL}$ of phosphate buffer, $\mathrm{pH} 7.4$ at $37^{\circ} \mathrm{C}$, under gentle shaking (60 rpm). The buffer was changed daily to stabilize $\mathrm{pH}$ and to maintain the solubility of released monomers constant. Samples were taken daily and dried under vacuum over phosphorus pentoxide as long as the matrix disks remained intact. The weight loss during erosion was determined from dried samples. For the determination of the erosion front position, dried samples were first fixed in a split specimen mount from Energy Beam Inc., Agawam, MA, which held the disks vertically in place up to the center. The brittle disks could then be broken in the middle by bending off the free half with flat tweezers. The created crosssections of fixed disk halves were gold coated and examined by scanning electron microscopy (SEM) using a Stereoscan 250 MK3 microscope from Cambridge Instruments, Cambridge, MA. Two parameters were measured: the height of the polymer matrix cylinder (i.e., the width of the cross-sections) and the distance between the polymer matrix surface and the erosion front that separates eroded from non-eroded polymer.

Calculations and Simulations. All algorithms for simulations and calculations were programmed in Pascal. Programs were run on a Macintosh IIsi from Apple Computer Inc., Cupertino, CA, using a Pascal compiler from Symantec, Cupertino, CA.

\section{Results and Discussion}

3.1. Investigation of Eroded Polymer Disk CrossSections. Changes of polymer microstructure were assessed from a time series of eroded polymer cross-sections. Figure 1 shows a time series of eroded p(CPP-SA) 20:80 disks. The single figures represent cutouts of cross-sections through eroded polymer matrices after different erosion times. The surfaces, which are in contact with water during erosion, are located to the left and right of the individual figures. The different patterns of the scale bars at the top of the figures indicate which areas of the matrices are eroded and which are not. The total thickness was determined by measuring the surface to surface distance from these SEM pictures. It was found that the thickness of samples does not change over a period of at least 6 days for $p(C P P-S A) ~ 20: 80$ and 12 days for $p(C P P-S A)$ 50:50.16 The erosion front which separates eroded from non-eroded polymer can best be seen after 3 days. Furthermore, this front appears parallel to the surface. The position of the erosion front was measured as the distance between front to the surface divided by half the total thickness of the individual disk.

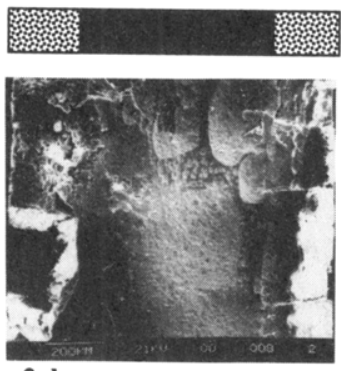

2 days

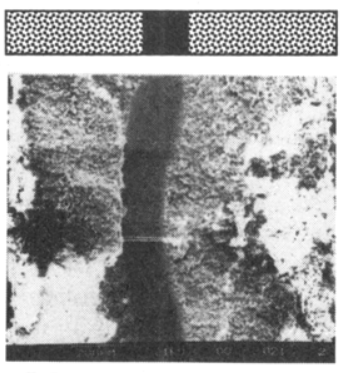

5 days

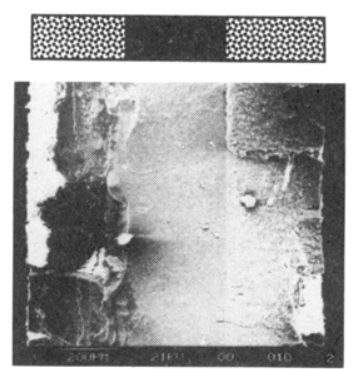

3 days

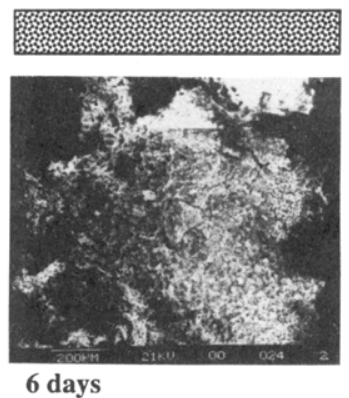

Figure 1. Time series of cross-sections through eroded matrices of p(CPP-SA) 20:80 captured by SEM. The stippled bar at the top of figure indicates eroded polymer; the solid bar indicates non-eroded polymer.

3.2. General Algorithm of the Model. In formulating a general algorithm, we first distinguished between composite and elementary events. The erosion of small parts of bulk polymer was assumed to be an elementary random event ${ }^{20}$ that could not be divided into simpler events. Other parameters, like the movement of the erosion front or the porosity of the systems, were assumed to be composite events that can be related to the erosion of small parts of the polymer matrix. To describe the overall erosion of a polymer, matrices were represented by a two-dimensional rectangular grid consisting of $n_{x} \times n_{y}$ grid points. Each grid point or pixel, $P_{i, j}\left(1<i<n_{x}, 1<j<n_{y}\right)$, refers to a part of the polymer matrix and represents either "sloweroding" or "fast-eroding" units. Rather than covering a complete matrix by this grid, it was sufficient to take only one cutout into account, as shown in Figure 2. Since the changes in the polymer upon erosion are symmetrical with respect to the middle of the matrix ${ }^{5}$, the grid needs to cover only half of a cross-section. Therefore, a reflecting boundary condition ${ }^{21}$ was imposed on the horizontal grid line at $j=n_{y}$ to account for the truncation of the grid in the middle of the matrix. To account for the limited area that is covered in the $x$ direction, periodic boundary conditions ${ }^{22}$ were imposed on the vertical grid lines at $i$ $=1$ and $i=n_{x}$. This connects the vertical borders of the grid and transforms it into a cylindrical surface. Erosion was simulated using the following algorithm:

1. The pixels on the computational grid were divided into two classes. They represent slow-eroding (or crystalline) areas and fast-eroding (or amorphous) areas. The ratio of the two species is, for example, determined by polymer crystallinity.

2. Polymer matrix pieces erode only after contact with an eroded neighbor. At $t=0$ the pixels $P_{i, 1}$ on the polymer matrix surface $j=1$ are in contact with buffer solution. They are, therefore, considered to have fictitious eroded neighbors.

3. After contact with an eroded neighbor, each pixel was assigned an individual life expectation, after which it is regarded eroded and removed from the grid.

4. The life expectation is a random variable, which is distributed according to a first-order Erlang distribution. ${ }^{23}$ 


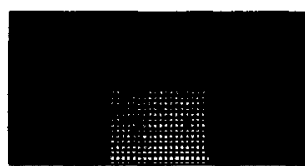

\section{polymer} matrix
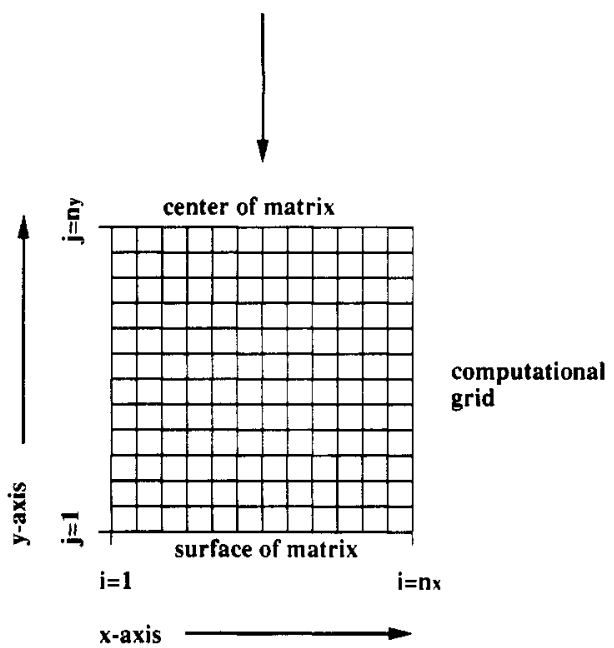

Figure 2. Representation of a polymer matrix by a rectangular computational grid.

Other distributions for describing life expectations could also potentially be used but were not investigated in this study.

5. The Erlang distribution constants are different for pixels representing slow- and fast-eroding areas.

The described algorithm was transformed in the next step into a set of formal mathematical expressions to run the model on a computer.

3.3. Configuring the Computational Grid Prior to Erosion. First, the way the grid describes the polymer matrix was defined. Therefore, a pixel $P_{i, j}$ was assumed to be in one of three possible states: fast-eroding (amorphous), slow-eroding (crystalline), or "eroded". Varible $x_{i, j}$ defines these possible states:

$$
\begin{array}{rlrl}
x_{i, j} & =1 & \text { "crystalline" pixel } \\
x_{i, j}=0 & \text { "amorphous" pixel } \\
x_{i, j}=-1 & \text { "eroded" pixel }
\end{array}
$$

At time $t=0$, the status $x_{i, j}$ of all pixels was assessed from consecutive Bernoulli trials. ${ }^{19}$ This yields a random distribution of fast- and slow-eroding areas on the grid representing the polymer matrix:

$$
\begin{gathered}
c\left(x_{i, j}\right)=1-\chi \quad x_{i, j}=0 \\
c\left(x_{i, j}\right)=\chi \quad x_{i, j}=1 \\
c\left(x_{i, j}\right)=0 \quad \text { all other values }
\end{gathered}
$$

where $\chi$ is the crystallinity of the system and $1 \leq i \leq n_{x}$, $1 \leq j \leq n_{y} . c\left(x_{i, j}\right)$ is the probability that a pixel $P_{i, j}$ represents a "crystalline" or an "amorphous" part of the polymer matrix. The expected value of $c\left(x_{i, j}\right)$ is $\chi .^{19}$ Figure 3 shows an example for a grid $n_{x}=n_{y}=100$ with $\chi=0.35$. Black pixels represent crystalline polymer areas, and white pixels amorphous polymer areas.

For computational reasons, we defined a function which describes the status of a pixel as either eroded or noneroded:

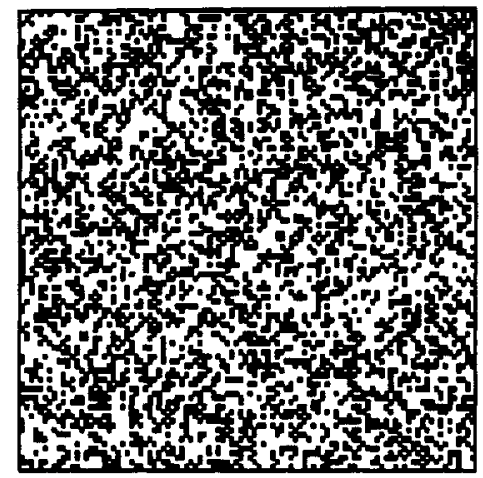

Figure 3. Example of a grid representing a matrix $\left(n_{x}=n_{y}=\right.$ $100, \chi=0.35$ ). Black pixels represent crystalline areas, and white pixels amorphous areas.

$$
\begin{aligned}
s\left(x_{i, j}\right)=1 & x_{i, j}=0 \\
s\left(x_{i, j}\right)=1 & x_{i, j}=1 \\
s\left(x_{i, j}\right)=0 & x_{i, j}=-1
\end{aligned}
$$

Before simulating matrix erosion, we defined the initial and boundary conditions. A hypothetical grid line of pixels $P_{i, 0}\left(1 \leq i \leq n_{x}\right)$ was added to the first row of pixels as an initial condition. The status of these pixels was the same as for eroded polymer and accounts for the fact that erosion begins at the polymer surface:

$$
x_{i, 0}=0 \text { and } s\left(x_{i, 0}\right)=0 \text { for } 1 \leq i \leq n_{x}
$$

The boundary conditions required the introduction of three hypothetical grid lines: $P_{0, j}, P_{n_{x}+1, j}\left(1 \leq j \leq n_{y}\right)$, and $P_{i, n_{y}+1}\left(1 \leq i \leq n_{x}\right)$ :

$$
\begin{array}{cc}
x_{i, n_{y}+1}=x_{i, n_{y}-1} & 1<i<n_{x} \\
x_{n_{x}+1, j}=x_{1, j} & 1<j<n_{y} \\
x_{0, j}=x_{n_{x} j} & 1<j<n_{y}
\end{array}
$$

Equation 4 represents the insulation condition for the grid line representing the center of the disk, while eq 5 represents the periodic boundary conditions which prevent finite size effects on the borders $i=1$ and $i=n_{x}$ of the grid.

3.4. Simulation of Erosion. After the grid had been set up the rules for the simulation of erosion had to be defined. It was assumed that the "erosion of a pixel" is a random event that can be described by a Poisson process of first order. ${ }^{23}$ The probability mass function for such a process is

$$
p=\lambda t \mathrm{e}^{-\lambda t} \quad t \geq 0
$$

where $p$ designates the probability by which a pixel "degrades" in the time interval $[0, t]$, where $t$ is the time. $\lambda$ is the average "arrival rate" of the process ${ }^{23}$ and can be considered a time constant. $\lambda$ is, therefore, greater for pixels representing amorphous areas $\left(\lambda_{a}\right)$ relative to crystalline ones $\left(\lambda_{c}\right)$. The probability that a pixel erodes at time $t$ is described by a first-order Erlang probability density function: ${ }^{23}$

$$
e(t)=\lambda e^{-\lambda t}
$$

where $t$ is a random variable representing the time interval from the appearance of the first eroded neighbor of a pixel until the moment of its own "erosion". $e(t)$ is the 
probability that a pixel degrades after time $t$. The expected value can be calculated from eq 6 by integration with respect to $t$ over the interval $[0, \infty]$ :

$$
e(t)_{\text {exp }}=1 / \lambda
$$

$e(t)_{\exp }$ is the average life expectation of a pixel after its first contact with an eroded neighbor. Unfortunately, the results using eq 7 depend on the grid size that is used to represent a matrix. This can be illustrated by the following example. If we represent a unit area of the polymer matrix by one pixel, the average life expectation of this unit area according to eq 8 is $1 / \lambda$. Representing the same area by two pixels, we can calculate the total average life expectation as the sum of two single values ${ }^{23}$ and obtain a value of $2 / \lambda$. Thus, the erosion of the same polymer area would take twice as much time if we increased the grid size by a factor of 2. To account for this size effect the parameter $\lambda$ had to be adjusted to account for the number of pixels in the $y$ direction. Substituting $\lambda n_{y}$ for $\lambda$ in eq 7 we find that size effects are now avoided:

$$
e_{n_{y}}(t)=\lambda n_{y} e^{-t \lambda n_{y}}
$$

Equation 9 allows simulations that are independent of the grid size. The equation could also be expanded to account for the number of eroded neighbors by introducing a factor in the exponent as well as before the exponential. This could be useful for future studies.

To introduce the randomness of erosion, life expectations have to be chosen randomly in such a way that they are distributed according to eq 9 . For the calculation of life expectations from eq 9 a sampling technique was used that sets the integral of eq 9 equal to $\epsilon$, a random variable equally distributed in the interval $[0,1]::^{24}$

$$
\epsilon=1-\mathrm{e}^{-t \lambda n_{y}}
$$

After rearranging eq 10 , we can calculate the life expectation of a pixel $P_{i, j}$ as a function of the random variable $\epsilon$.

$$
t_{i, j}=\frac{1}{\lambda n_{y}} \ln (1-\epsilon)
$$

To account for fast- and slow-eroding areas, different values, $\lambda_{\mathrm{a}}$ and $\lambda_{\mathrm{c}}$, have to be used for $\lambda$. Equation 11 is now used for the calculation of life expectations, which are distributed according to a first-order Erlang distribution, by simply generating random variables of type $\epsilon$ on the computer. The time $t_{i, j}$ at which a pixel $P_{i, j}$ "erodes" is calculated according to

$$
\begin{gathered}
t_{i, j}=t_{\min }(i, j)+\frac{1}{\lambda_{\mathrm{c}} n_{y}} \ln (1-\epsilon), x_{i, j}=1, \sum_{i=1}^{i+1} \sum_{j-1}^{j+1} s\left(x_{i, j}\right)<9 \\
t_{i, j}=t_{\min }(i, j)+\frac{1}{\lambda_{\mathrm{a}} n_{y}} \ln (1-\epsilon), x_{i, j}=0, \sum_{i=1}^{i+1} \sum_{j-1}^{j+1} s\left(x_{i, j}\right)<9 \\
t_{i, j}=t_{i, j} \quad x_{i, j}=-1, \sum_{i=1}^{i+1} \sum_{j=1}^{j+1} s\left(x_{i, j}\right)<9 \\
t_{i, j}=\infty \quad \sum_{i=1}^{i+1} \sum_{j=1}^{j+1} s\left(x_{i, j}\right)=9
\end{gathered}
$$

where $\lambda_{c}$ and $\lambda_{a}$ are the constants for amorphous and crystalline pixels and $t_{\min }(i, j)$ is the time at which a pixel had its first contact with an eroded neighbor. For the calculation of the lifetime $t_{i, 1}$ of pixels on the surface,

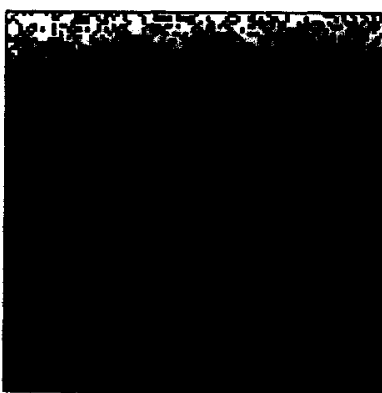

$\mathrm{t} * \lambda \mathrm{a}=0.042$

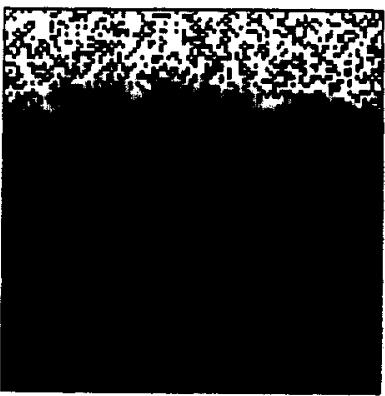

$\mathrm{t} * \lambda \mathrm{a}=0.104$

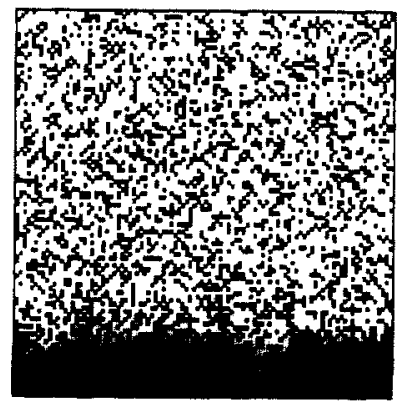

$\mathrm{t} * \lambda_{\mathrm{a}}=0.339$

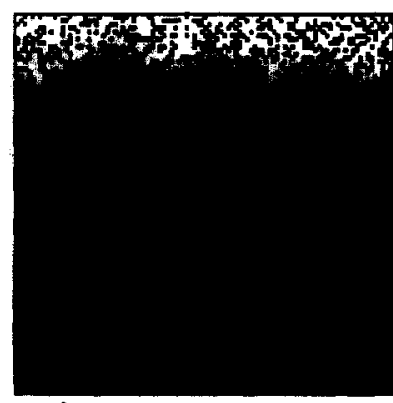

$t * \lambda \mathrm{a}=0.074$

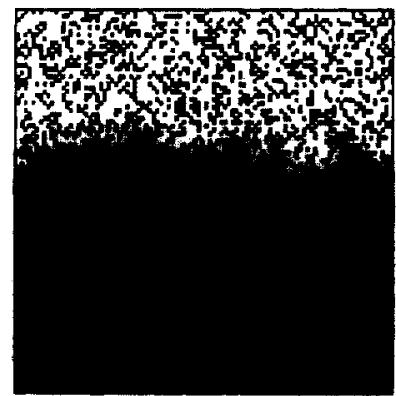

$\mathrm{t} * \lambda_{\mathrm{a}}=0.157$

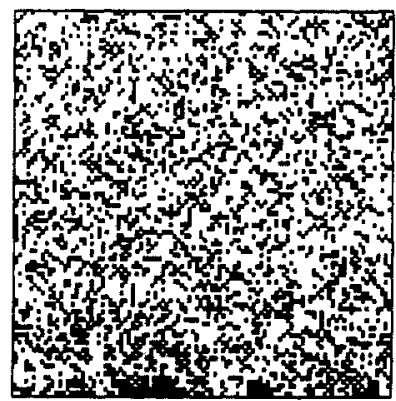

$\mathrm{t} * \lambda_{\mathrm{a}}=0.396$
Figure 4. Time series of simulated erosion of a polymer matrix $\left(n_{x}=n_{y}=100, \chi=0.35, \lambda_{c}=10^{-8} \mathrm{~s}^{-1}, \lambda_{\mathrm{a}}=10^{-6} \mathrm{~s}^{-1}\right)$.

$t_{\min }(i, j)$ was set to 0 . After the calculation of the life expectation for surface pixels, erosion proceeds by the determination of the pixel $P_{i, j}$ with the shortest lifetime $t_{i, j} . t_{\min }$ is set to the value of this minimum, the status of the "eroded" pixel is changed to $x_{i j}=-1$, and the function $s\left(x_{i, j}\right)$ is updated accordingly. The function $t_{i, j}$ is then used for the calculation of life expectations for those pixels which are now newly in contact with an eroded neighbor.

A time series of matrices that was obtained when the procedure was applied to the grid shown in Figure 3 can be seen in Figure 4. The surface of the represented polymer matrix is located at the top of the cutouts. The bottom of the individual pictures designates the middle of the matrix. Obviously, erosion starts from the surface of the grid and moves toward the center. The contiguous dark area represents the non-eroded polymer, whereas the white area represents eroded polymer. The erosion front that separates both zones is obviously parallel to the surface of the grid, which is in good agreement with experimental results (cf. Figure 1). The dark pixels in the eroded area represent polymer that has not yet eroded despite being passed by the erosion front. Most of these pixels represent crystalline polymer areas and have thus a higher chance of resisting erosion. By expanding the grid into three dimensions, we can assume that these remainders form an interconnected network which is mechanically stable but highly porous. Figure 4 shows that even after long erosion times there are still pixels in the uppermost line on the grid, where the surface of the non-eroded matrix was 


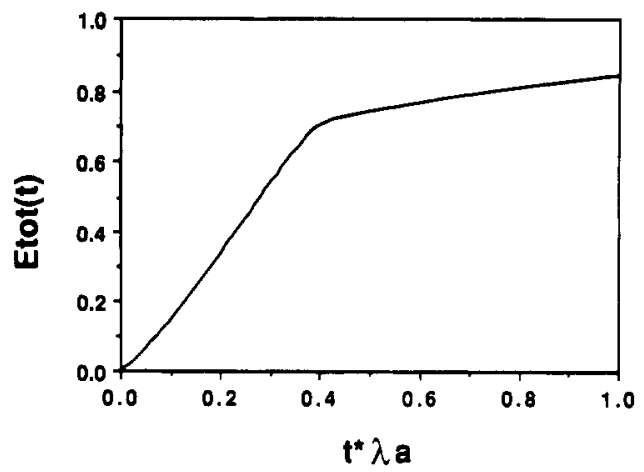

Figure 5. Changes in total porosity during erosion $\left(n_{x}=n_{y}=\right.$ $\left.100, x=0.35, \lambda_{c}=10^{-8} \mathrm{~s}^{-1}, \lambda_{1}=10^{-6} \mathrm{~s}^{-1}\right)$.

located originally. This shows that the total thickness of the represented polymer matrix has not changed during the simulation.

3.5. Functions for the Evaluation of Results. After qualitative agreement of the model predictions shown in Figure 4 with experimental findings of Figure 1 was attained, it was necessary to evaluate the simulation results quantitatively. For that purpose several parameters were defined that can be calculated from the computational grid during the simulation of erosion.

The total porosity is an important parameter as it is also experimentally measurable by mercury intrusion porosimetry. ${ }^{16}$ Its value was calculated from simulations by counting the relative number of non-eroded pixels from values $s\left(\mathbf{x}_{i, j}\right)$. From this sum the total porosity can be calculated by dividing by the total number of grid points and subtracting from 1 :

$$
E_{\mathrm{tot}}(t)=1-\frac{1}{n_{x} n_{y}} \sum_{i=1}^{n_{z}} \sum_{j=1}^{n_{y}} s\left(x_{i, j}\right)
$$

Figure 5 shows the change of total porosity with time during the simulation shown in Figure 4. In an initial phase, the changes are rapid due to the erosion of fast-eroding pixels. Once the erosion front has reached the center of the disk, there remain only slow-eroding pixels which slow down the increase in porosity.

In addition to the average porosity throughout the matrix, the porosity distribution along the $y$ axis perpendicular to the surface can be calculated. This is achieved by counting the number of non-eroded pixels per position $j$, subtracting it from the total number of pixels, $n_{x}$, per grid line, and dividing by the total number of pixels $n_{x}$ :

$$
E(j, t)=1-\frac{1}{n_{x}} \sum_{i=1}^{n_{x}} s\left(x_{i, j}\right) \quad 1 \leq j \leq n_{y}
$$

For any time $t$ and position $j$ along the direction of erosion front movement, the porosity can be calculated. Figure 6 shows profiles at different times for the simulations shown in Figure 4. They give good insight into the local changes of porosity inside the matrix. All profiles are similar and consist of three major parts. In the area close to the surface, porosity is almost uniformly distributed, which is due to long-surviving crystalline parts of the polymer. When the erosion front is approached, the porosity profiles drop in a sigmoidal manner, and beyond the erosion front the porosity is zero.

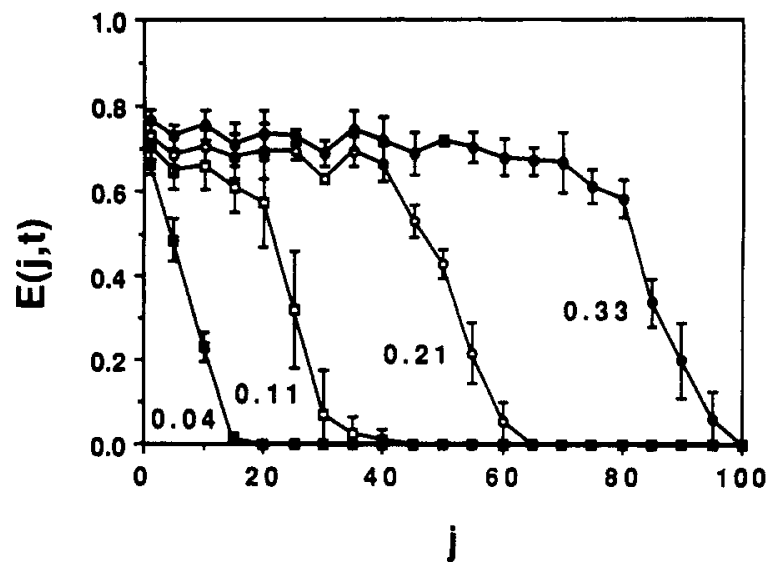

Figure 6. Distribution of porosity in a slab perpendicular to its surface during erosion for various times $t \lambda_{\mathrm{a}}\left(n_{x}=n_{y}=100, \chi=\right.$ $\left.0.35, \lambda_{c}=10^{-8} \mathrm{~s}^{-1}, \lambda_{\mathrm{a}}=10^{-6} \mathrm{~s}^{-1}\right)$.

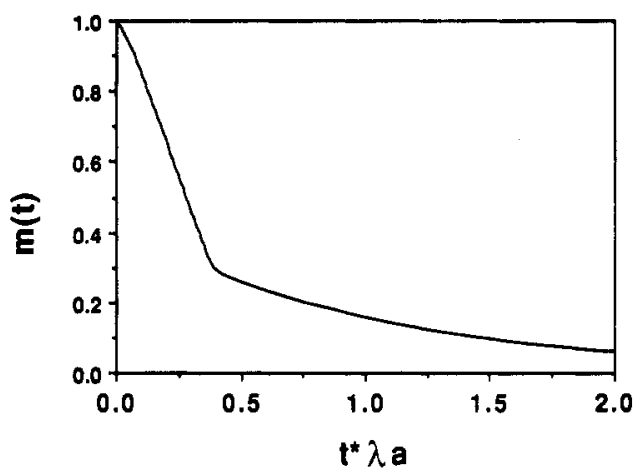

Figure 7. Fraction of non-eroded polymer during erosion $\left(n_{x}=\right.$ $\left.n_{y}=100, \chi=0.35, \lambda_{c}=10^{-8} \mathrm{~s}^{-1}, \lambda_{\mathrm{a}}=10^{-6} \mathrm{~s}^{-1}\right)$.

The remaining relative mass of non-eroded polymer during erosion can be calculated from the total porosity:

$$
m(t)=1-E_{\text {tot }}(t)
$$

The results for the simulation shown in Figure 4 can be seen in Figure 7. The profile again displays a biphasic process which shows a fast drop in weight before the erosion front has reached the center of the matrix and which slows down after it. Since the loss of weight can be measured experimentally very accurately, it was one of the functions that was used for the determination of erosion rate constants by curve fitting.

Characteristic for the group of polymers that was investigated is the movement of the erosion front that separates eroded from non-eroded polymer. ${ }^{16}$ We calculated the average position of the erosion front from the simulations. For each column $i$, the position $f_{i}$ of the foremost eroded pixel was determined. This yielded $n_{x}$ minima from which the erosion front position was calculated as an average value:

$$
f(t)=\frac{1}{n_{x}} \sum_{i=0}^{n_{x}} f_{i}(t)
$$

Figure 8 shows the results from the simulations shown in Figures 1 and 2. The sigmoidal profile shows a slight lag at the beginning and becomes linear with time. Together with the weight loss the movement of the erosion front was used for the determination of erosion rate constants.

3.6. Evaluation of Experimental Data. Quantitative evaluations were accomplished by fitting simulated to experimental data, which required the repetitive simulation of erosion. To save computer time, it was necessary to reduce the grid size to an extent that was small enough 


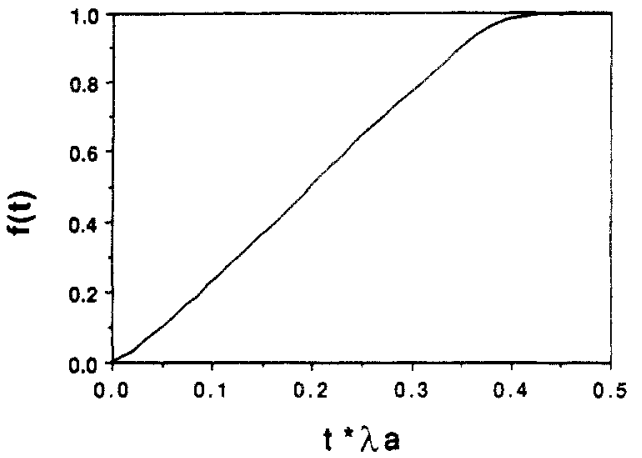

Figure 8. Position of the erosion front during erosion $\left(n_{x}=n_{y}\right.$ $=100, \chi=0.35, \lambda_{c}=10^{-8} \mathrm{~s}^{-1}, \lambda_{\mathrm{a}}=10^{-6} \mathrm{~s}^{-1}$.

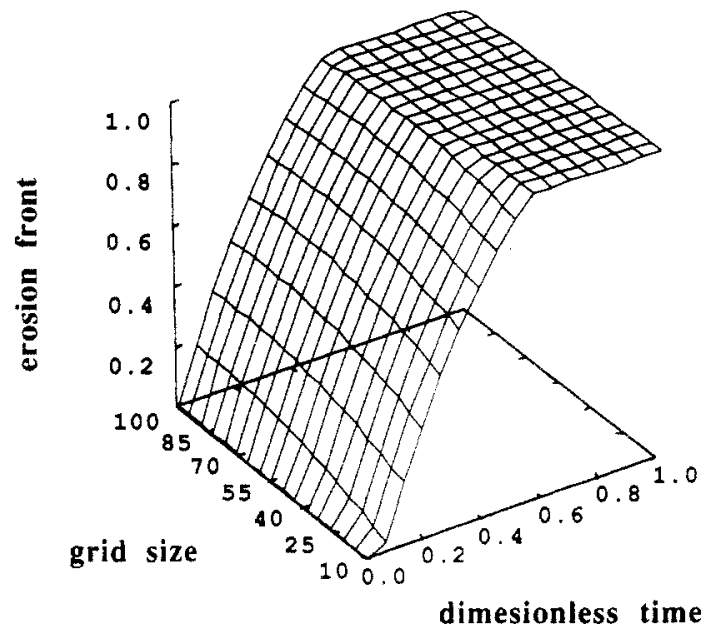

Figure 9. Influence of the grid size on the function describing the erosion front position $\left(\lambda_{\mathrm{c}}=10^{-8} \mathrm{~s}^{-1}, \lambda_{\mathrm{a}}=10^{-6} \mathrm{~s}^{-1}\right)$.

to have reasonable computer run times but yet accurate enough with respect to the results. Therefore, it was necessary to investigate the influence of the grid size parameters $n_{x}$ and $n_{y}$ on the simulated results. First, values of $\lambda_{c}\left(10^{-8} \mathrm{~s}^{-1}\right)$ and $\lambda_{\mathrm{a}}\left(10^{-6} \mathrm{~s}^{-1}\right)$ were guessed and $a$ crystallinity $\chi=0.35$ was chosen, which is a realistic value for p(CPP-SA) 20:80. ${ }^{25}$ The influence of the grid size parameters $n_{x}$ and $n_{y}$ on the movement of the erosion front described by eq 16 was investigated. Simulations were conducted for various values of $n_{y}$ with $n_{x}=n_{y}$ to investigate differences in the movement of the erosion front for large and small grid sizes. Figure 9 shows how the profile for the erosion front movement, calculated from eq 16, changes when the grid size is increased from 10 to 100. It can be seen that even for small grid sizes there are no substantial changes in the shape of the erosion front movement profiles. We concluded that grid sizes of $n_{y}=$ 100 would be sufficient for all subsequent investigations. In a second study we wanted to evaluate what the influence of the grid size $n_{x}$ is while keeping $n_{y}$ constant at 100 . We varied $n_{x}$ between 10 and 100 . Figure 10 shows that for $n_{x}$ larger than 20 there are almost no changes in the profile for the erosion front movement. From these preliminary experiments we concluded that a grid size of $n_{x}=50, n_{y}$ $=100$ would be ideal for further calculations in order to have reasonable run time on the computer and reasonable accuracy.

Using the grid size parameters mentioned above, we determined the model constants $\lambda_{c}$ and $\lambda_{a}$ for both polymers. The functions for the movement of the erosion front (eq 16) and the change of the polymer matrix mass (eq 15) were fit simultaneously to the experimental data given in Tables I and II. Using the modified simplex

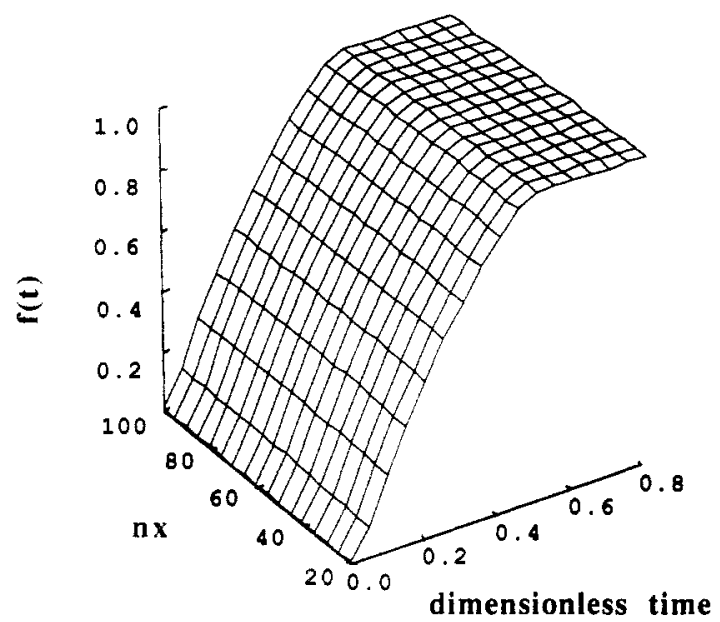

Figure 10. Influence of the ratio $n_{x} / n_{y}$ on the function describing the erosion front position $\left(\lambda_{c}=10^{-8} \mathrm{~s}^{-1}, \lambda_{\mathrm{a}}=10^{-6} \mathrm{~s}^{-1}\right)$.

Table I. Experimental Data for the Movement of the Erosion Front, $f(t)$, and the Change of Mass, $m(t)$, for p(CPP-SA) 20:80

\begin{tabular}{cll}
\hline time (days) & \multicolumn{1}{c}{$f(t)$} & \multicolumn{1}{c}{$m(t)$} \\
\hline 0 & 0 & 1 \\
1.02 & $0.20 \pm 0.01$ & $0.93 \pm 0.01$ \\
1.93 & $0.41 \pm 0.03$ & $0.81 \pm 0.01$ \\
3.02 & $0.55 \pm 0.05$ & $0.66 \pm 0.02$ \\
4.03 & $0.67 \pm 0.04$ & $0.55 \pm 0.03$ \\
4.93 & $0.77 \pm 0.09$ & $0.43 \pm 0.04$ \\
5.93 & $0.93 \pm 0.03$ & $0.27 \pm 0.07$
\end{tabular}

Table II. Experimental Data for the Movement of the Erosion Front, $f(t)$, and the Change of Mass, $m(t)$, for p(CPP-SA) 50:50

\begin{tabular}{cll}
\hline time (days) & \multicolumn{1}{c}{$f(t)$} & \multicolumn{1}{c}{$m(t)$} \\
\hline 0 & 0 & 1 \\
0.99 & $0.13 \pm 0.02$ & $0.93 \pm 0.01$ \\
1.97 & $0.22 \pm 0.03$ & $0.84 \pm 0.02$ \\
4.03 & $0.45 \pm 0.03$ & $0.67 \pm 0.03$ \\
6.01 & $0.87 \pm 0.04$ & $0.50 \pm 0.03$ \\
8.04 & $0.84 \pm 0.01$ & $0.37 \pm 0.09$ \\
9.99 & $0.87 \pm 0.04$ & $0.33 \pm 0.09$ \\
12.02 & 1 & $0.27 \pm 0.02$
\end{tabular}

Table III. Model Constants $\lambda_{c}$ and $\lambda_{e}$ for the Erosion of p(CPP-SA) 20:80 and p(CPP-SA) 50:50 Determined from Experimental Data

\begin{tabular}{lll}
\hline & \multicolumn{1}{c}{$\lambda_{\mathrm{a}}\left(\mathrm{s}^{-1}\right)$} & \multicolumn{1}{c}{$\lambda_{\mathrm{c}}\left(\mathrm{s}^{-1}\right)$} \\
\hline p(CPP-SA) $20: 80$ & $7.32 \times 10^{-7}$ & $8.75 \times 10^{-9}$ \\
p(CPP-SA) $50: 50$ & $2.7 \times 10^{-7}$ & $3.85 \times 10^{-11}$
\end{tabular}

method ${ }^{26}$ of Nelder and Mead, ${ }^{27}$ we varied the parameters $\lambda_{c}$ and $\lambda_{a}$ until the sum of the squared differences between the experimental and simulated data was minimized. To account for the random distribution of crystallinity and the randomness of the erosion of single pixels, each run for a set of data $\lambda_{c}$ and $\lambda_{a}$ was repeated five times using five different sets of random number sequences.

The values $\lambda_{c}$ and $\lambda_{a}$ for both polymers are given in Table III. The graphical result of the best fit for $p$ (CPPSA) 20:80 can be seen in Figure 11. There is good agreement between experimental and theoretical data. For the second polymer, $p(\mathrm{CPP}-\mathrm{SA})$ 50:50, the theoretical result shown in Figure 12 is still in agreement with experimental data. The increased deviations between experimental and simulated data are mainly due to the accumulation of crystallized monomers in the porous network created during erosion (a process not accounted for by the current model) which is caused by the low solubility of CPP monomer. ${ }^{16}$ The weight loss predicted 


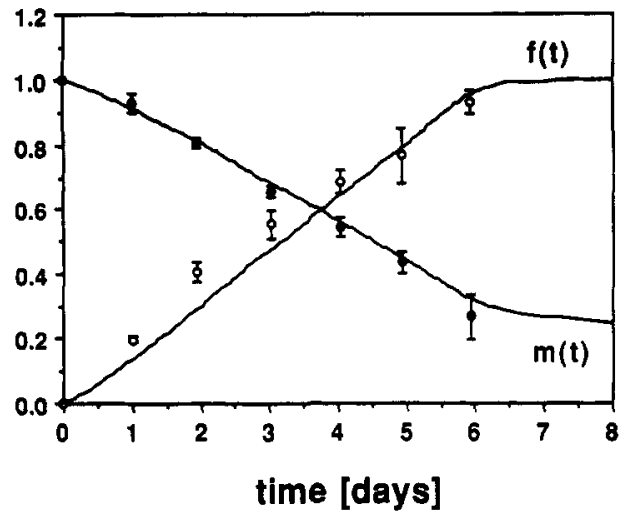

Figure 11. Fit of the simulated erosion front position and the remaining mass to experimental $p(C P P-S A) 20: 80$ data $\left(n_{x}=50\right.$, $n_{y}=100, \chi=0.35$ ).

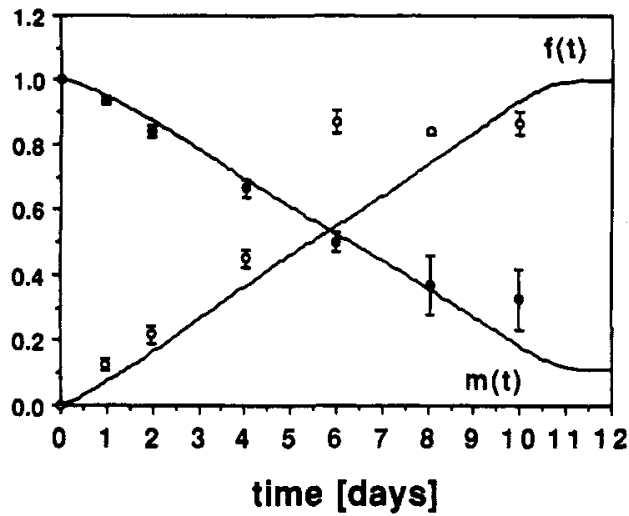

Figure 12. Fit of the simulated erosion fron position and the remaining mass to experimental $\mathrm{p}(\mathrm{CPP}-\mathrm{SA})$ 50:50 data $\left(n_{x}=50\right.$, $n_{y}=100, \chi=0.1$ ).

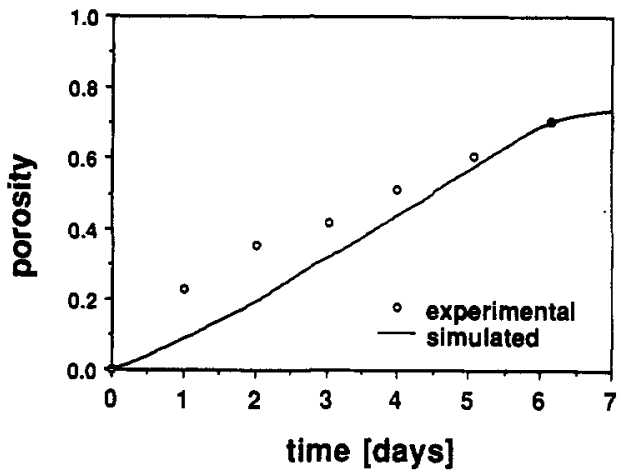

Figure 13. Predicted and experimentally determined porosity of p(CPP-SA) 20:80 polymer disks $\left(n_{x}=50, n_{y}=100, \chi=0.35\right)$.

by the model is, therefore, higher than that experimentally determined.

To demonstrate the model's potential for predicting data, the degradation rate constants in Table III were used for prediction of polymer matrix porosity during erosion. ${ }^{16}$ Figures 13 and 14 show the proposed model is useful for the prediction of data that have not been used for the determination of rate constants.

\section{Conclusions}

The erosion of polyanhydride matrices was successfully modeled with a Monte Carlo simulation that calculates the life expectation of small pieces of polymer matrix from a first-order Erlang distribution. There is good agreement between experimental and theoretical data (cf. Figures 11 and 12). The obtained data for $\lambda_{c}$ and $\lambda_{\mathrm{a}}$ given in Table III mirror the different erosion velocities for both polymers. p(CPP-SA) 50:50 erodes more slowly than p(CPP-SA)

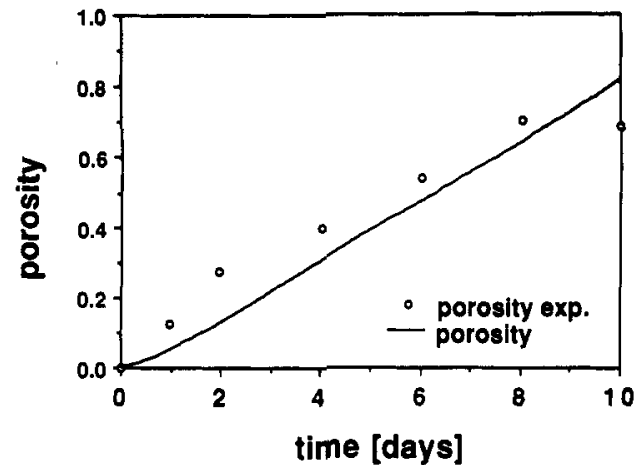

Figure 14. Predicted and experimentally determined porosity of $\mathrm{p}(\mathrm{CPP}-\mathrm{SA})$ 50:50 polymer disks $\left(n_{x}=50, n_{y}=100, \chi=0.1\right)$.

$20: 80^{28}$ and has consequently lower values for both constants. Once the polymer degradation constants were known, the prediction of erosion parameters such as porosity (cf. Figures 13 and 14) was possible. The present model becomes, therefore, a useful tool in predicting the erosion behavior of polymers.

Acknowledgment. Special thanks are due the Deutsche Forschungsgemeinschaft (Grant GO 565/1-1), Bonn, FRG, and the National Institutes of Health (Grant CA 52857), which funded this project. We also want to thank Scios-Nova Pharmaceuticals, Baltimore, MD, for providing the polymers.

\section{Abbreviations}

$c\left(x_{i, j}\right) \quad$ probability by which a pixel $P_{i, j}$ represents a "crystalline" or an "amorphous" part of the polymer

$\chi \quad$ crystallinity of the polymer

$e(t) \quad$ first-order Erlang probability density function describing the distribution of life expectations of a pixel after contact with an eroded neighbor

$e_{\text {exp }} \quad$ average life expectation of a pixel after contact with an eroded neighbor(s)

$e_{n_{y}}(t) \quad$ first-order Erlang probability density function describing the distribution of life expectations of pixels depending on a specific grid size $n_{y}$

$E(j, t) \quad$ porosity in the $j$ th layer of the grid

$E_{\text {tot }}(t)$ total porosity

$\epsilon$ random variable which is equally distributed in $[0,1]$

$f\left({ }^{*} \mathrm{t}\right) \quad$ average position of the erosion front at time $t$ $f_{i} \quad$ position of the erosion front in the $i$ th layer

$\lambda$ rate constant $\left(\mathrm{s}^{-1}\right)$

$\lambda_{c} \quad$ rate constant for the degradation of crystalline polymer areas $\left(\mathrm{s}^{-1}\right)$

$\lambda_{a} \quad$ rate constant for the degradation of amorphous polymer areas $\left(\mathrm{s}^{-1}\right)$

$m(t)$ relative mass of non-eroded polymer at time $t$

$n_{x} \quad$ number of pixels in the $x$ direction

$n_{y} \quad$ number of pixels in the $y$ direction

$p \quad$ probability mass function for a first-order Poisson process

$P_{i, j} \quad$ pixel on the computational grid at $i, j$

$s\left(x_{i, j}\right) \quad$ function defining the status of a pixel as either eroded or non-eroded

$t \quad$ time (s) 
$t_{i, j} \quad$ time after the start of the experiment at which pixel $P_{i, j}$ degrades (s)

$t_{\min }(i, j)$ time after which pixels $P_{i, j}(i=-1,1 ; j=-1,1)$ have an additional eroded neighbor (s)

$x_{i, j} \quad$ random variable defining the status of a pixel as crystalline, amorphous, or eroded

\section{References and Notes}

(1) Leenslang, J. W.; Pennings, A. J.; Ruud, R. M.; Rozema, F. R.; Boering, G. Resorbable materials of poly(L-lactide). VI. Plates and screws for internal fracture fixation. Biomaterials 1987,8 , $70-73$.

(2) Brem, H.; Mahaley, M. S.; Vick, N. A.; Black, K. L.; Schold, S. C.; Burger, P. C.; Friedman, A. H.; Ciric, I. S.; Eller, T. W. Cozzens, J. W.; Kenally, J. N. Interstitial chemotherapy with drug polymer implants for the treatment of recurrent gliomas. $J$. Neurosurg. 1991, 74, 441-446.

(3) Penuelas, J. Degradable plastics. AQEIC Bol. Tec. 1992, 43 (2), 75-78.

(4) Li, C.; Kohn, J. Synthesis of poly(iminocarbonates): degradable polymers with potential application as disposable plastics and as biomaterials. Macromolecules 1989, 22 (5), 2029-2036.

(5) Tamada, J.; Langer, R. Erosion mechanism of hydrolytically degradable polymers. Proc. Natl. Acad. Sci. U.S.A. 1993, 90, $552-556$.

(6) Fan, L. T.; Singh, S. K. Controlled Release-A Quantitative Treatment; Springer-Verlag: Berlin, 1989.

(7) Rosen, H. B.; Kohn, J.; Leong, K.; Langer, R. Bioerodible polymers for controlled release systems. In Controlled Release Systems: Fabrication Technology; Hsieh, D., Ed.; CRC Press: Boca Raton, FL, 1988; Vol. II, pp 83-110.

(8) Cooney, D. O. Effect of geometry on the dissolution of pharmaceutical tablets and other solids: surface detachment kinetics controlling. AIChE J. 1972, 18 (2), 446-449.

(9) Baker, R. W.; Lonsdale, H. K. Erodible controlled release systems. Org. Coat. Plast. Chem. 1976, 3, 229.

(10) Heller, J.; Baker, R. W. Theory and practice of controlled drug delivery from bioerodible polymers. In Controlled Release of Bioactive Materials; Baker, R. W., Ed.; Academic Press: New York, 1980.

(11) Thombre, A. G.; Himmelstein, K. J. Modelling of drug release kinetics from laminated device having an erodible drug reservoir. Biomaterials 1984, 5, 250-254.

(12) Lee, P. I. Diffusional release of a solute from a polymeric matrix - approximate analytical solutions. J.Membr.Sci.1980, $7,255-275$.
(13) Thombre, A. G.; Himmelstein, K. J. A simultaneous transportreaction model for controlled drug delivery from catalyzed bioerodible polymer matrices. AIChE J. 1985, 31 (5), 759-766.

(14) Zygourakis, K. Discrete simulations and bioerodible controlled release systems. Polym. Prepr. (Am. Chem. Soc., Div. Polym. Chem.) 1989, 30 (1), 456-457.

(15) Zygourakis, K. Development and temporal evolution of erosion fronts in bioerodible controlled release devices. Chem. Eng. Sci. 1990, 45 (8), 2359-2366.

(16) Göpferich, A.; Langer, R. The influence of microstructure and monomer properties on the erosion mechanism of a class of polyanhydrides. J. Polym. Sci., in press.

(17) Domb, A.; Ron, E.; Langer, R. Polyanhydrides. Encyclopedia of Polymer Science and Engineering, Supplement Volume, 2nd ed.; Wiley: New York, 1989; pp 648-665.

(18) Tamada, J.; Langer, R. The development of polyanhydrides for drug delivery applications. J. Biomater. Sci., Polym. Ed. 1992, $3(4), 315-353$.

(19) Boas, M. Mathematical Methods in the Physical Sciences, 2nd ed.; Wiley: New York, 1983; pp 685-739.

(20) Kalos, M. H.; Whitlock, P. A. Monte Carlo Methods; Wiley: New York, 1986; p 7.

(21) Habermann, R. Elementary and Applied Partial Differential Equations, 2nd ed.; Prentice-Hall: Englewood Cliffs, NJ, 1987; pp 10-12.

(22) Heermann, D.W. Computer Simulation Methods in Theoretical Physics, 2nd ed.; Springer-Verlag: Berlin, 1990; pp 15-16.

(23) Drake, A. W. Fundamentals of Applied Probability Theory; McGraw-Hill: New York, 1988; pp 129-144.

(24) Cashwell, E. D.; Everett, C. J. A Practical Manual on the Monte Carlo Method for Random Walk Problems; Pergamon Press: New York, 1959; pp 4-10.

(25) Mathiowitz, E.; Ron, E.; Mathiowitz, G.; Amato, C.; Langer, R. Morphological Characterization of Bioerodible Polymers. 1. Crystallinity of Polyanhydride Copolymers. Macromolecules 1990, 23, 3212-3218.

(26) Spendley, W.; Hext, G. R.; Himswoth, F. R. Sequential application of simplex designs in optimization and evolutionary operation. Technometrics 1962, 4 (4), 441-461.

(27) Nelder, J. A.; Mead, R. A simplex method for function minimization. Comput. J. 1964, 7, 308-313.

(28) Leong, K. W.; Brott, B. C.; Langer, R. Bioerodible polyanhydrides as drug-carrier matrices. I: Characterization, degradation, and release characteristics. J. Biomed. Mater. Res. 1985, $19,941-955$. 